\title{
Das Stigma von Suchterkrankungen verstehen und überwinden
}

\section{Understanding and Overcoming the Stigma of Addiction}

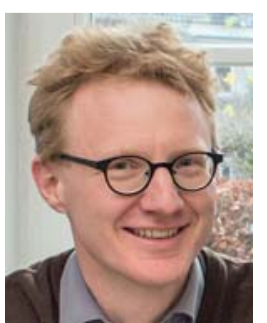

Georg Schomerus

\author{
Autor \\ Georg Schomerus \\ Institut \\ Klinik und Poliklinik für Psychiatrie und Psychotherapie, \\ Universitätsmedizin Greifswald \\ Bibliografie \\ DOI https://doi.org/10.1055/s-0043-109924 \\ Psychiat Prax 2017; 44: 249-251 \\ (c) Georg Thieme Verlag KG Stuttgart · New York \\ ISSN 0303-4259 \\ Korrespondenzadresse \\ Prof. Dr. Georg Schomerus, Klinik und Poliklinik für \\ Psychiatrie und Psychotherapie, Universitätsmedizin \\ Greifswald, Ellernholzstraße 2, 17475 Greifswald \\ georg.schomerus@uni-greifswald.de
}

„Das Stigma von Suchterkrankungen verstehen und überwinden" war das Thema einer Klausurtagung, zu der sich im September 2016 eine Gruppe von Suchtexperten in Greifswald traf. Eine Woche lang arbeiteten 14 Wissenschaftler, Betroffene und Therapeuten mit unterschiedlichsten Fach- und Erfahrungshorizonten daran, ein Memorandum zu erstellen, das aufzeigen soll, wie wir dem Stigma von Suchterkrankungen entgegentreten können. Die Tagung wurde vom Bundesministerium für Gesundheit gefördert. In diesem Editorial möchte ich aus psychiatrischer Sicht die wichtigsten Punkte des Memorandums [1] aufgreifen und der Frage nachgehen, welche Implikationen sich daraus für den Umgang mit Suchtkrankheiten in der Psychiatrie ergeben.

\section{Das Stigma von Suchtkrankheiten im Fokus}

Das Stigma von Suchterkrankungen ist in den letzten Jahren stärker in den Fokus geraten - zunächst, um ein gravierendes Defizit festzustellen: Während es buchstäblich Tausende von Arbeiten über das Stigma anderer psychischer Krankheiten wie Depression oder Schizophrenie gibt, wurde das Stigma von Substanzabhängigkeiten wissenschaftlich lange vernachlässigt [2]. Aber nicht nur wissenschaftlich, sondern auch praktisch wurde das Stigma von Suchterkrankungen bisher nur zaghaft angegangen. Im Gegensatz zu anderen psychischen Krankheiten gab es bisher kaum Anti-Stigma-Initiativen im Zusammenhang mit Sucht, und die bestehenden Initiativen hatten es schwer, eine größere Öffentlichkeit herzustellen, obwohl offen- sichtlich gerade Suchtkrankheiten besonders stark stigmatisiert werden [3]. Aktuell wird deshalb versucht, Wissensbestände aus der Forschung über das Stigma psychischer Krankheiten für den Umgang mit dem Suchtstigma nutzbar zu machen [4]. Allerdings hat dieser Transfer Grenzen, bestimmte Strategien der Entstigmatisierung von psychischen Krankheiten scheinen für Suchtkrankheiten ungeeignet zu sein [5]. Auch wenn es viele falsche Stereotype über Menschen mit Suchtkrankheiten gibt, wird die Korrektur bestimmter Stereotype nur begrenzt Erfolg haben, weil es zu viele Situationen gibt, in denen sie zutreffen. So hängen zum Beispiel Gewalt und Alkoholintoxikation unbestreitbar eng zusammen, auch wenn selbstverständlich nicht alle Menschen mit Alkoholproblemen gewalttätig sind. Auch Unberechenbarkeit oder sozial unangepasstes Verhalten sind gängige Symptome des Substanzgebrauchs. Substanzabhängigkeit und -konsum stellen vielfach ein unerwünschtes Verhalten dar, insbesondere wenn dabei ein Kontrollverlust auftritt und bestimmte soziale Normen verletzt werden. Eine Verharmlosung des Konsums und der daraus resultierenden gesundheitlichen und sozialen Probleme kann keine glaubwürdige Anti-Stigma-Strategie sein.

\section{Gesellschaftliche Legitimität des Suchtstigmas}

Vermutlich ist aber auch die gesellschaftliche Legitimität des Suchtstigmas höher als bei anderen stigmatisierten Gruppen. Die Ausgrenzung von Menschen mit Suchtkrankheiten kann als soziales Signal verstanden werden, dass das Suchtverhalten 
nicht toleriert wird. Sie hat dann einen quasi erzieherischen Zweck - Menschen mit Suchtproblemen werden schlecht behandelt, damit sie die Notwendigkeit spüren, ihr Verhalten zu ändern. Das Stigma erscheint vermutlich vielen Menschen als legitime Antwort auf Suchtprobleme. Diese mehr oder weniger explizit geäußerte Zustimmung zur Ausgrenzung macht die Entstigmatisierung von Suchterkrankungen deutlich schwerer und könnte die geringe öffentliche Resonanz auf bisherige Kampagnen zur Entstigmatisierung von Suchterkrankungen erklären.

\section{Durch Stigmatisierung können Suchtprobleme nicht gelöst werden}

Tatsächlich zeigen zahlreiche Untersuchungen, dass Stigmatisierung und Ausgrenzung keine gute Lösung für Suchtprobleme sind, sondern die Probleme im Gegenteil verstärken [6]. Das Memorandum konstatiert, dass Stigmatisierung die Behandlung von Suchtproblemen erschwert, die negativen Folgen der Krankheit verstärkt und die Betroffenen und Angehörigen isoliert, anstatt sie zu unterstützen. Dabei trifft das Stigma vor allem schwer betroffene Menschen sowie Menschen aus ohnehin schon benachteiligten Gruppen.

\section{Strategien gegen Stigma}

Wie kann das Stigma von Suchtkrankheiten reduziert werden? Die Autoren des Memorandums kommen zu dem Schluss, dass Entstigmatisierung nur dann funktionieren kann, wenn ein besserer, alternativer Umgang mit Suchtkrankheiten aufgezeigt wird. Hilfe statt Ausgrenzung, Befähigung statt Abwertung müssen im Mittelpunkt eines stigmafreien Umgangs mit Suchtkrankheiten stehen. Die Empfehlungen, die das Memorandum in den Bereichen „Qualitative Verbesserung im Hilfesystem und bei der Prävention“, „Befähigung (Empowerment)“, „Kommunikation und Koordination“, „Forschung“ und „Konzeptionelle und rechtliche Weiterentwicklungen“ gibt, beschreiben Schritte auf dem Weg zu einem besseren, stigmafreien Umgang mit Suchtkrankheiten.

\section{Verbesserungen im Hilfesystem}

Dabei richtet das Memorandum den Blick auch auf die Stigmatisierung von Menschen mit Suchtkrankheiten innerhalb des Behandlungssystems. Intensiv wurde auf der Tagung zum Beispiel die Frage der Diagnosestellung diskutiert. Es wurde kritisiert, dass F1-Diagnosen an einer Person „kleben“, auch wenn die Person schon viele Jahre abstinent gelebt hat. Im Memorandum werden deshalb Anforderungen an ein nicht-stigmatisierendes Krankheitskonzept von Suchtkrankheiten formuliert: Dieses müsse z.B. auf das Kontinuum psychischer Krankheit und Gesundheit Bezug nehmen [7], und Diagnosen dürften nicht lebenslang an den Menschen haften, sondern sollten Menschen so lange begleiten wie sie für sie nützlich sind.

Es ist immer leichter, Diskriminierung in anderen Disziplinen und Strukturen zu entdecken als in der eigenen Profession, aber auch innerhalb der Psychiatrie gibt es Regeln und Abläufe, die zum Nachteil von Patienten mit „F1-Diagnose“ wirken. So muss man kritisch fragen, inwieweit das durchaus noch verbreitete Vorgehen angemessen ist, vor der (psychotherapeutischen) Behandlung einer komorbiden Angststörung, PTBS oder Depression eine Zeit der Abstinenz zu fordern. Pragmatisch wäre Nüchternheit zur Therapie völlig ausreichend, was der Tatsache Rechnung tragen würde, dass häufig zunächst die Komorbidität behandelt werden muss, bevor eine dauerhafte Abstinenz möglich ist. Analog muss man auch fragen, ob nicht die Ausgliederung der Behandlung von Menschen mit Suchtkrankheiten in spezialisierte Kliniken oder auf spezialisierte Stationen einer guten Behandlung zuwiderläuft, weil eine notwendige gleichzeitige Behandlung psychiatrischer Komorbiditäten damit erschwert wird. Über die Psychiatrie hinaus weist die Forderung des Memorandums die Separierung der Hilfesysteme zu überwinden. Hier zielt das Memorandum auf die traditionelle Aufteilung der Hilfesysteme in Suchthilfe, medizinische/psychiatrische Hilfe und Psychotherapie ab, die dazu führt, dass Menschen mit Abhängigkeitserkrankungen häufig somatisch und psychotherapeutisch schlechter behandelt werden als Menschen mit anderen Erkrankungen, auch wenn sowohl die somatische als auch die psychiatrische Komorbidität bei Suchterkrankungen sehr hoch ist. Gleichzeitig nimmt das Memorandum auch auf Entwicklungen bei der Behandlung von Abhängigkeitserkrankungen Bezug, die bereits jetzt einer Stigmatisierung entgegenwirken, wie das Motivational Interviewing (motivierende Gesprächsführung) und der Community Reinforcement Approach (Gemeindeorientierte Suchttherapie).

\section{Eine Frage der Haltung}

Die größte Herausforderung für eine stigmafreie Behandlung von Menschen mit Abhängigkeitserkrankungen stellt aber sicher die Haltung der Beschäftigten im Gesundheitswesen dar, nicht nur in der Psychiatrie. Zu häufig werden Abhängigkeitserkrankungen als Ausdruck persönlichen Fehlverhaltens angesehen, und zu häufig ist der Umgang mit den Betroffenen von Missfallen, Herablassung und moralischer Überheblichkeit geprägt. Im Memorandum wird deutlich gemacht, dass es sich hier nicht nur in Bezug auf den Behandlungserfolg um einen höchst fragwürdigen Ansatz handelt, sondern auch um ein ethisches Problem und eine Frage der Menschenwürde. So lange sich Menschen mit Abhängigkeitserkrankungen auf diese Weise schlecht behandelt fühlen, werden sie nachvollziehbarerweise professionelle Hilfe, wenn irgend möglich, vermeiden. Das Memorandum fordert, dass Anti-Stigma-Kompetenz Teil der Ausbildung aller Gesundheitsberufe wird [8], um frühzeitig eine angemessene therapeutische Haltung gegenüber Menschen mit Suchtkrankheiten zu fördern. Dabei müsste vermittelt werden, dass Ausgrenzung und Herabsetzung das genaue Gegenteil dessen erreichen, was eigentlich intendiert ist.

Insgesamt zeigt das Memorandum, dass das Stigma von Suchterkrankungen keine Randerscheinung auf dem Gebiet der Suchterkrankungen ist, sondern ein zentrales Phänomen, das unseren Umgang mit den Betroffenen und ihren Erkrankungen maßgeblich beeinflusst. Das Stigma von Suchtkrankheiten zu überwinden, ist deshalb eine zentrale Herausforderung für 
einen guten Umgang mit Suchtkrankheiten, nicht nur in der Öffentlichkeit, sondern auch innerhalb des Hilfesystems.

Interessenkonflikt

Der Autor gibt an, dass kein Interessenkonflikt besteht. Gefördert vom Bundesministerium für Gesundheit (Zuwendung des Bundes, Kapitel 1504, Titel 68405).

Literatur

[1] Schomerus G, Bauch A, Elger B et al. Memorandum - Das Stigma von Suchtkrankheiten verstehen und überwinden. 2017: Im Internet: http://www.dg-sucht.de/fileadmin/user_upload/pdf/aktuelles/Me morandum_Stigmatisierung.pdf (Stand: 26.4.2017)

[2] Schomerus G. Warum werden Menschen mit Alkoholabhängigkeit in besonderer Weise stigamtisiert, und was kann man dagegen tun? Psychiat Prax 2011; 38: 109-110

[3] Schomerus G, Lucht M, Holzinger A et al. The Stigma of Alcohol Dependence Compared with Other Mental Disorders: A Review of Population Studies. Alcohol Alcohol 2011; 46: 105-112

[4] Corrigan PW, Schomerus G, Shuman V et al. Developing a research agenda for reducing the stigma of addictions, part II: Lessons from the mental health stigma literature. Am J Addict 2017; 26: 67-74

[5] Schomerus G, Finzen A. Probleme und Implikationen der Einschätzung des Gewaltrisikos von psychisch kranken Menschen. Psychiat Prax 2016; 43: 355-356

[6] Glass JE, Williams EC, Bucholz KK. Psychiatric comorbidity and perceived alcohol stigma in a nationally representative sample of individuals with DSM-5 alcohol use disorder. Alcohol Clin Exp Res 2014; 38: $1697-1705$

[7] Schomerus G, Angermeyer MC, Baumeister SE et al. An online intervention using information on the mental health-mental illness continuum to reduce stigma. European psychiatry: the journal of the Association of European Psychiatrists 2016; 32: 21 - 27

[8] Arnaud N, Diestelkamp S, Wartberg L et al. Nachhaltiger Transfer des Gesundheitsnetz Alkohol im Jugenalter: Eine Kooperation aus Forschung, Praxis und Politik. Psychiat Prax 2015; 42 (Suppl. 01): S35S38 